\title{
Contemporary Management of Patients on Warfarin, Aspirin And Clopidogrel Requiring Dentoalveolar Surgery
}

\author{
Sunil Sidana ${ }^{1}$, Jyotsna Galinde ${ }^{2}$
}

\begin{abstract}
Patients with a variety of medical conditions often receive aspirin, warfarin or clopidogrel to prevent complications from atrial fibrillation, thromboembolisms or stroke. Although these medications can be lifesaving; it also can put patients at greater risk of experiencing haemorrhage after dental surgery. Therefore, a decision must be made whether to interrupt or continue anticoagulant treatment in patients undergoing various dental procedures. We have presented a review of this topic, including a brief description of the more commonly encountered anticoagulant and antiplatelet medications and the current recommendations for the surgical management of these patients.
\end{abstract}

Key Words: Aspirin, warfarin, clopidogrel, dental surgery, extraction, INR

In dentoalveolar surgery, the management of patients who are on aspirin or clopidogrel or warfarin is varied, controversial and frequently anecdote based ${ }^{1}$. Dental clinicians are frequently faced with the question of whether to continue or stop these medications before extraction. Even a significant disparity exists on the same issue among the oral and maxillofacial surgeons who are not up-to-date with the current literature ${ }^{2}$. Some dentists believe that consulting with patients' physicians is the solution to the problem of what to do for patients receiving continuous anticoagulant therapy. It has become common for the practitioner to stop these medications without being aware of the evidence of benefit or risks associated with stopping these medications. "Simply following a physician's order" is unlikely to be an effective legal defence if the dentist is charged with failure to exercise reasonable professional judgment ${ }^{3}$. Current evidence based guidelines with regards to management of patients on these medications is as follows.

\section{Warfarin}

Patients can require warfarin for various diagnoses, such as atrial fibrillation (AF), pulmonary embolism, myocardial infarction, stroke, deep venous thrombosis(DVT), and antiphospholipid syndrome, or

1 Reader

2 Professor \& Head

Dept. of Oral \& Maxillofacial Surgery

MGM Dental College \& Hospital, Navi Mumbai

Address for Correspondence

Reader, Dept. of Orthodontics

MGM Dental College

Kamothe, Navi Mumbai 410209

Mob: 9820798821

Email: srcindia@hotmail.com because they have prosthetic heart valves ${ }^{4-7}$. As a vitamin $K$ antagonist, warfarin decreases the coagulation of blood. The anticoagulant effect of warfarin takes 48-72 hours to develop fully, with an estimated duration of action of 2 to 5 days and a reported half-life of 2.5 days $^{8}$. Thromboembolic events are known to occur when warfarin is discontinued in the perioperative period ${ }^{9-11}$. Management of patients receiving long term oral anticoagulation who require dental extraction is based on an assessment of risks: the risk of procedure related bleeding if anticoagulants are continued measured against the thromboembolic risks if anticoagulants are stopped.

Thromboembolic events are associated with considerable morbidity and mortality. Permanent disability or death occurs in:

- $70 \%$ to $75 \%$ of patients who experience an arterial thromboembolism (e.g. stroke, myocardial infarction, pulmonary embolism),

- $4 \%$ to $10 \%$ of patients who have a venous thromboembolism (e.g. deep vein thrombosis).

Patients with heart valve replacements or recurrent thromboembolism (INR target between 3.0 and 4.0) are at the highest risk for serious thromboembolic events if their anticoagulant therapy is temporarily stopped or decreased.

The risk of thromboembolic events if warfarin is discontinued appears to vary from 0.02 to $1 \%$.

Continuing warfarin during dental surgical procedures will increase the risk of postoperative bleeding requiring intervention. Stopping warfarin is no guarantee that the risk of postoperative bleeding requiring intervention will be eliminated 
as serious bleeding can occur in non-anticoagulated patients. Most cases of postoperative bleeding can be managed by pressure or repacking and suturing the socket. The incidence of postoperative bleeding not controlled by local measures varies from $0 \%$ to $3.8 \%$.

Bleeding complications while inconvenient do not carry the same risks as thromboembolic complications.

The activity of warfarin is expressed using the international normalised ratio (INR). For an individual not taking warfarin a normal coagulation profile is an INR of 1.0.

Published trial data suggests that minor dental surgical procedures can be safely carried out on patients with an INR $<\mathbf{4}^{12}$. The consensus from reviews on the management of dental patients taking warfarin is that minor dental surgical procedures should be carried out without alteration to the patient's warfarin therapy if the INR is within the therapeutic range(INR 2.0 to 4.0$)^{1-15}$.

The INR should be measured prior to dental procedures, ideally within 24 hours before the procedure. For patients who have a stable INR, an INR measured within 72 hours before the procedure is acceptable. Patients presenting with an INR much higher than their normal value, even if it is less than 4.0 should have their procedure postponed and should be referred back to clinician maintaining their anticoagulant therapy.

\section{Antiplatelet Therapy}

Antiplatelet therapy has been shown to be effective in decreasing the risk of myocardial infarction and nonfatal stroke among patients with peripheral vascular disease. In contrast, discontinuation of this therapy in high-risk patients has been shown to increase the risk of cardiac complications and death $^{16-18}$. Various medications have been used as antiplatelet therapy. The more common plateletinhibiting medications include aspirin, clopidogrel, ticlopidine, and dipyridamole.

\section{Aspirin}

Aspirin, acetylsalicylic acid, is a nonsteroidal antiinflammatory drug that exhibits analgesic, antipyretic, anti-inflammatory, and antiplatelet properties. Aspirin has been shown to be powerful secondary prevention agent, reducing the risk of myocardial infarction and ischemic stroke by up to $20 \%$ in patients diagnosed with cardiovascular disease ${ }^{19}$. Its mechanism of action involves an irreversible inhibition of the activity of cyclooxygenase- 1 and a modification of the enzymatic activity of cyclooxygenase-2. Cyclooxygenase is an enzyme responsible for the conversion of arachidonic acid to prostaglandins, prostacyclin and thromboxane. The irreversible nature of the inhibition of cyclooxygenase is unique to aspirin among its counterparts.

\section{Clopidogrel}

Clopidogrel is an antiplatelet drug with a mechanism of action causing irreversible inhibition of an adenosine diphosphate receptor important in promoting platelet aggregation and cross-linking of platelets by fibrin. Clopidogrel is used alone or in combination with aspirin as both have synergistic effect

Aspirin begins irreversibly inhibiting platelet aggregation within one hour of ingestion and clopidogrel within two hours; this lasts for the life of the platelets (7-10 days). The effect is only overcome by the manufacture of new platelets. Complete recovery of platelet aggregation may occur in $50 \%$ of cases by day three and in $80 \%$ of cases by day four.

Platelet function is commonly assessed using the cutaneous bleeding time test. One study has found that the cutaneous bleeding time test has no role in the prediction of bleeding in the dental setting ${ }^{20}$. The cutaneous bleeding time test should not be used to estimate the haemorrhagic risk in a patient on antiplatelet medication. There is currently no suitable bioassay test sophisticated enough to be used routinely for the monitoring of side effects associated with antiplatelet medications.

Aspirin can double the baseline bleeding time but this may still be within or just outside the normal range. It has been reported that only $20 \%$ to $25 \%$ of patients using aspirin have an abnormal bleeding time. Clopidogrel is considered a more potent antiplatelet agent and can prolong the bleeding time by 1.5 to 3 times normal ${ }^{21,22}$. Sensitivity to antiplatelet agents varies from one person to another.

Stroke and myocardial infarction have been associated with cessation of antiplatelet medication approximately 10 days before the event. Stopping aspirin prior to surgical procedures may increase the risk of thromboembolic events by $0.005 \%{ }^{11}$.

Patients taking antiplatelet medications will have a prolonged bleeding time but this may not be clinically relevant. Postoperative bleeding after dental procedures can be controlled using local haemostatic measures in patients taking antiplatelet monotherapy (one antiplatelet agent).

There is insufficient evidence to comment on the bleeding risk if patients take both aspirin and clopidogrel. 
Patients are more at risk of permanent disability or death if they stop antiplatelet medication prior to a surgical procedure than if they continue it.

Published reviews of the available literature advise that antiplatelet monotherapy should not be stopped prior to dental surgical procedures.

There is insufficient evidence to comment if patients take both aspirin and clopidogrel.

\section{When patients are taking dual antiplatelet therapy either their interventional cardiologist should be contacted for advice or the patient should be referred to a dental hospital or hospital-based oral/ maxillofacial surgeon.}

Planned surgery should ideally be

1. At the beginning of the day- this allows more time to deal with immediate re-bleeding problems.

2. Early in the week- this allows for delayed rebleeding episodes occurring after 24-48 hours to be dealt with during the working week.

3. A local anaesthetic containing vasoconstrictor should be administered by infiltration or by intraligamentary injection wherever practical.

4. Sockets should be gently packed with an absorbable hemostatic dressing e.g. oxidised cellulose (surgical), or absorbable gelatin sponge (gelfoam), and then carefully sutured. Hemostatic dressings promote and stabilise clot formation by providing a mechanical matrix. Following closure pressure should be applied to the socket(s) by using a gauze pad that patient bites down on for 20 minutes.

5. Efforts should be made to make the procedure as a traumatic as possible and any bleeding should be managed using local measures.

6. For postoperative pain control, generally paracetamol is considered the safest simple analgesic for patients taking warfarin and it may be taken in normal doses if pain control is needed and no contraindication exists.

7. Metronidazole interacts with warfarin and should be avoided wherever possible.

8. Avoid prescribing aspirin and NSAIDs.

9. Tranexamic acid is an antifibrinolytic agent that inhibits the breakdown of fibrin clots. Its primary action is to block the binding of plasminogen and plasmin to fibrin therefore preventing fibrinolysis. It has been used in anticoagulated dental patients as a local hemostatic agent in the form of mouthwash. When used alone with no local hemostatic dressing, tranexamic acid mouthwash reduces postoperative bleeding compared to placebo mouthwash. When used with local hemostatic dressing and suturing, tranexamic acid mouthwash provides little additional reduction in postoperative bleeding ${ }^{23-26}$. $4.8 \%$ concentration solution of tranexamic acid mouthwash is commonly used.

\section{Summary}

1. The consensus from reviews on the management of dental patients taking warfarin is that patients requiring dental surgical procedures in primary care and who have an International Normalised Ratio (INR) below 4.0 should continue warfarin therapy without dose adjustment.

2. Most cases of postoperative bleeding are easily treated with local measures such as packing with a haemostatic dressing, suturing and pressure.

3. Published reviews of the available literature advise that antiplatelet monotherapy should not be stopped prior to dental surgical procedures.

\section{References:}

1. Dodson T: No need to routinely discontinue anticoagulants before dental extractions. Evidence-Based Dentistry (2002) $3,100 \pm 101$

2. Ward B, Smith M: Dentoalveolar procedures for the anticoagulated patient: Literature recommendations versus current practice. J Oral Maxillofac Surg 65:1454, 2007

3. American Dental Association, American Academy of Orthopaedic Surgeons. Antibiotic prophylaxis for dental patients with total joint replacements. JADA 1997; 128:1004-8.

4. Sear JW, Higham H: Issues in the perioperative management of the elderly patient with cardiovascular disease. Drugs Aging 19:429, 2002

5. Cunningham LL Jr, Brandt MT, Aldridge E: Perioperative treatment of the patient taking anticoagulation medication. Oral Maxillofac Surg Clin North Am 18:151, 2006

6. Ananthasubramaniam K, Beattie JN, Rosman HS, et al: How safely and for how long can warfarin therapy be withheld in prosthetic heart valve patients hospitalized with a major hemorrhage? Chest 119:478, 2001

7. Solomon JM, Schow SR: The potential risks, complications, and prevention of deep vein thrombosis in oral and maxillofacial surgery patients. J Oral Maxillofac Surg $53: 1441,1995$

8. Aldridge E, Cunningham L: Current Thoughts on Treatment of Patients Receiving Anticoagulation Therapy. J Oral Maxillofac Surg 68:2879-2887, 2010

9. Wahl MJ. Dental surgery in anticoagulated patients. Arch Intern Med 1998; 158: 1610-6.

10. Todd DW. Anticoagulated patients and oral surgery. Arch Intern Med 2003; 163: 1242.

11. Kovich $\mathrm{O}$ and Otley CC. Thrombotic complications related to discontinuation of warfarin and aspirin therapy preoperatively for cutaneous operation. J Am Acad Dermatol 2003; 48: 233-7.

12. Evans IL, Sayers MS, Gibbons AJ, Price G, Snooks H, Sugar AW. Can warfarin be continued during dental extraction? Results of a randomised controlled trial. Br J Oral Maxillofacial Surg 2002; 40:248 \pm 242

13. Wahl MJ. Myths of dental surgery in patients receiving anticoagulant therapy. J Am Dent Assoc 2000; 131: 77-81.

14. Campbell JH, Alvarado F and Murray RA. Anticoagulation and minor oral surgery: should the anticoagulation regimen be altered? J Oral Maxillofac Surg 2000; 131: 131-5. 
15. Devani P, Lavery KM and Howell CJT. Dental extractions in patients on warfarin: is alteration of anticoagulant regime necessary? Br J Oral Maxillofac Surg 1998; 36: 107-11

16. Robless P, Mikhailidis DP, Stansby G: Systematic review of antiplatelet therapy for the prevention of myocardial infarction, stroke or vascular death in patients with peripheral vascular disease. Br J Surg 88:787, 2001

17. Sharma AK, Ajani AE, Hamwi SM, et al: Major noncardiac surgery following coronary stenting: When is it safe to operate? Catheter Cardiovasc Interv 63:141, 2004

18. Kapetanakis EL, Medlam DA, Boyce SW, et al: Clopidogrel administration prior to coronary artery bypass grafting surgery: The cardiologist's panacea or the surgeon's headache? EurHeart J 26:576, 2005

19. Lewis HD, Davis JW, Archibald DG, et al: Protective effects of aspirin against acute myocardial infarction and death in men with unstable angina: Results of a veteran's administration cooperative study. N Engl J Med 309:396, 1983

20. Shalom A and Wong L. Outcome of aspirin use during excision of cutaneous lesions. Ann Plast Surg 2003; 50: 296-8.

21. Harder S, Klinkhardt U and Alvarez JM. Avoidance of bleeding during surgery in patients receiving anticoagulant and/or antiplatelet therapy. Pharmacokinetic and pharmacodynamic considerations. Clin Pharmacokinet 2004; 43: 963-81.

22. Madan GA et al. Minor oral surgery without stopping daily low-dose aspirin therapy: A study of 51 patients. J Oral Maxillofac Surg 2005; 63: 1262-5.

23. Sindet-PedersonS,Ramström G, Bernvil S and Blombäck M. Hemostatic effect of tranexamic acid mouthwash in anticoagulant-treated patients undergoing oral surgery. $\mathrm{N}$ Engl J Med 1989; 320: 840-3.

24. Zanon E, Martinelli F, Bacci C, Cordioli GP and Girolami A. Safety of dental extraction among consecutive patients on oral anticoagulant treatment managed using a specific dental management protocol. Blood Coagul Fibrinolysis 2003; 14: 27-30.

25. Blinder D, Manor Y, Martinowitz U and Taicher S. Dental extractions in patients maintained on continued oral anticoagulant, comparison of local hemostatic modalities. Oral Surg Oral Med Oral Pathol Oral Radiol Endod 1999; 88: $137-40$.

26. Blinder D, Manor Y, Martinowitz U and Taicher S. Dental extractions in patients maintained on oral anticoagulant therapy: Comparison of INR value with occurrence of postoperative bleeding. Int J Oral Maxillofac Surg 2001; 30: 518-21. 